\title{
Measurement of train driver's brain activity by functional near-infrared spectroscopy (fNIRS)
}

\author{
T. Kojima ${ }^{1}$, H. Tsunashima ${ }^{2} \&$ T. Y. Shiozawa ${ }^{3}$ \\ ${ }^{1}$ Graduate School of Nihon University, Japan \\ ${ }^{2}$ College of Industrial Technology, Nihon University, Japan \\ ${ }^{3}$ School of Medicine, Nihon University, Japan
}

\begin{abstract}
Train drivers are strongly urged to avoid making human errors of judgment. To avoid human error in train operation, driving support systems should be developed in consideration of human behavior. In developing such systems it is important to understand the relation between the train operation and the brain activity of driver. The brain activity during the driving operation with a traindriving simulator is measured by a functional near-infrared spectroscopy (fNIRS). To compare the changes in the brain activity with the driving operation, we developed an analysis method that can extract the brain activity related to task by using a wavelet-based multi-resolution analysis. The results show that the brain function due to a train driving can be evaluated with the proposed method.

Keywords: railway, human factors, brain function, near-infrared spectroscopy, wavelet, multi-resolution analysis, train simulator.
\end{abstract}

\section{Introduction}

One of the characteristics of train driving is that the driving operation tends to be more monotonous than car driving. This decrease in the driver's arousal level may cause human error. Railway companies provide education and training to train crews as part of measures to prevent such human-error-related accidents. In addition, companies have introduced backup systems such as Automatic Train Stop (ATS) devices. On some railways, Automatic Train Operation (ATO) devices have been introduced to automate train operation. 
However, this increased automation requires train drivers to focus on monitoring tasks and may thus cause a worrying decrease in driver arousal levels. In the automotive field, drowsy driver detection and warning systems, which can detect drowsy driving on the basis of information about the driver's physiological state and/or driving behavior and give a warning, have been studied. Basic studies on human behavior have been conducted in the field of train operation, but these studies have not yet actively contributed to train operation support. To avoid human error in train operation, we consider that the development of driving support systems should take human behavioral characteristics into account.

In developing such systems, it is important to understand: 1) how train drivers recognize information, 2) what judgments they make on the basis of this information, and 3) what actions they take. A driving simulator that can measure the driver's physiological state or the like under various conditions is needed to analyze the driver's actions.

This paper describes the measurement of brain function during train operation using a functional near-infrared spectroscopy (fNIRS), and proposes a new analysis method for extracting the brain activity related to a driving operation by wavelet-based multi-resolution analysis.

\section{Development of train-driving simulator for evaluation of human factors}

For human factor studies, a train simulator is needed which can simulate realistic train operation. We developed a train-driving simulator which can change the environment flexibly to suit the purpose of the experiment.

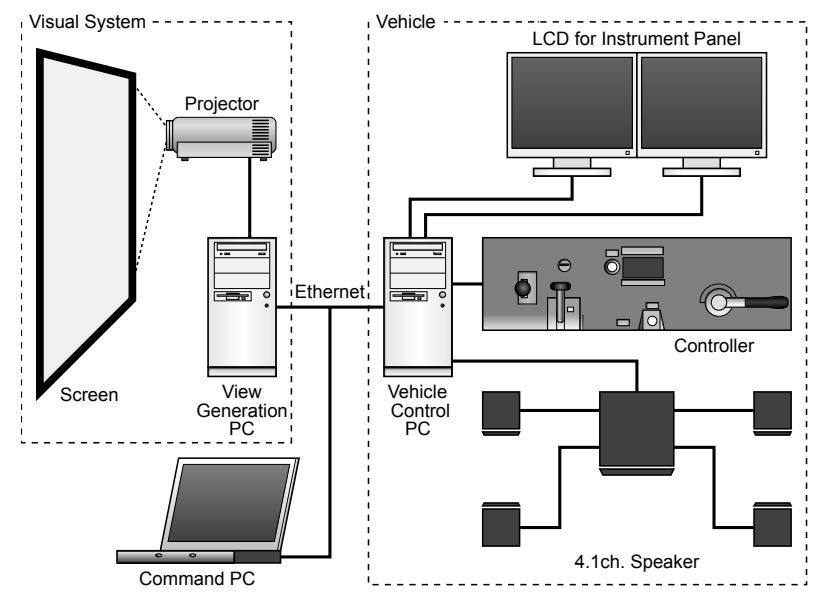

Figure 1: System configuration of train-driving simulator.

Fig. 1 shows the system configuration of the driving simulator that was developed. The simulator consists of three units: 1) a vehicle unit, composed of a 
vehicle control computer, a train cab controller, monitors for instrument panel, and speakers; 2) a visual system unit, composed of a view generation computer, a projector, and a screen; and 3) a command computer. Once the handle positions were input to the train control computer, actions such as the calculation of train movement, the generation of sounds, and the creation of instrument panel images on the monitor were carried out on the basis of the train's properties. When the calculated train position data were sent to the view generation computer, computer graphic view images were created and the images were projected by the projector onto the screen located at the front of the cab. The command computer could perform a number of actions: for example, it could cause accidents to occur and change stations.

\section{Measurement of brain function}

\subsection{Recent trends in brain function examination and the need for measurement during driving operations}

If we are to understand the mechanism of a potential accident, we need to study the brain activity of the person who might cause the accident, as representative of the person's physiological condition. Functional magnetic resonance imaging (fMRI) is attracting attention as a noninvasive method of diagnostic imaging. fMRI examination, which uses magnetism to enable the observation of localized changes in oxygenated hemoglobin levels in the brain, has contributed greatly to the clarification of brain functions such as language and cognition. However, fMRI has various limitations and is difficult to use in the evaluation of driving operations. For example, the examinee has to lie supine in a narrow cylinder, without moving his/her head during the examination.

In contrast, near-infrared spectroscopy (NIRS) has recently become popular. This is also noninvasive examination method. A relatively small type of NIRS with a single channel is has been applied to measurements during airplane operation [5]. This single channel NIRS can be used to observe the circulatory dynamics of only a small area in the brain. Functional near-infrared spectroscopy (fNIRS) [3, 4, 6], so-called optical topography, allows the mapping of wide area and evaluation of functional localization in the cerebrum using NIRS technology.

Either type of NIRS apparatus is characterized by the stable capture of circulatory dynamics in the brain during body movement in real time and promises to be useful for such evaluations.

\subsection{Principle of near-infrared spectroscopic measurement}

Fig. 2 shows a diagram of near-infrared spectroscopic measurement of cerebral blood flow. Near-infrared rays enter human tissues through optical fibers, propagate in the tissues and radiate from the tissues. NIRS detects the radiated rays, and measures relative variations of oxygenated hemoglobin (oxy-Hb) and deoxygenated hemoglobin (deoxy-Hb) based on those absorbencies. 


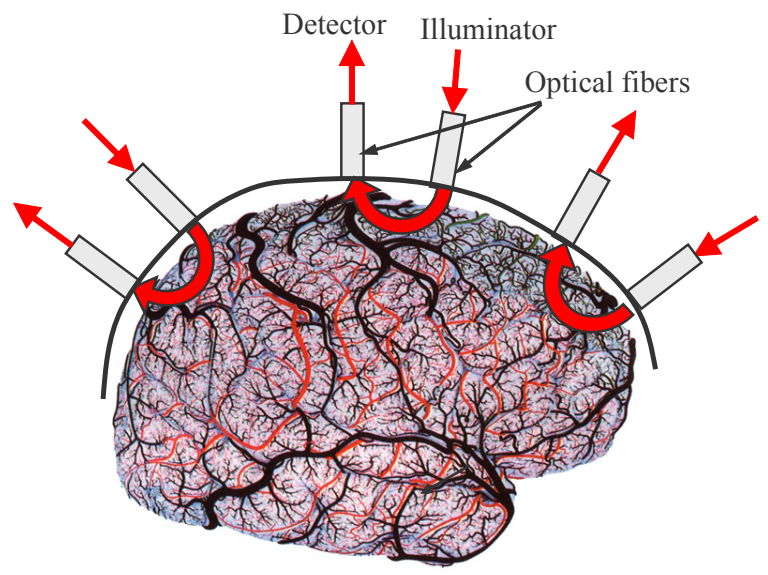

Figure 2: Diagram of near-infrared spectroscopic measurement.

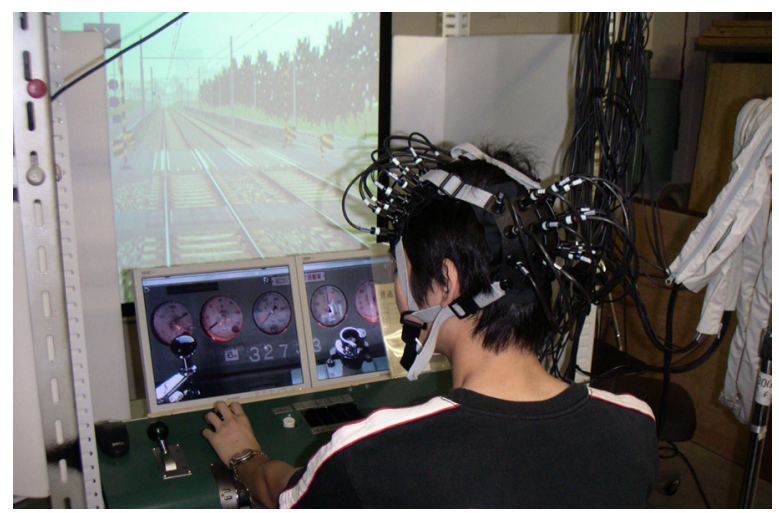

Figure 3: Experiment of measurement of brain function using train-driving simulator.

\subsection{Measurement of brain function using the train-driving simulator}

\subsubsection{Experimental methods}

We measured brain activity using the train-driving simulator. Fig. 3 shows how the experiment was carried out. We simulated a length of $2.3 \mathrm{~km}$ with three stations on a real railway line. A station-stop as long as $50 \mathrm{~s}$ was set in order to measure changes in cerebral blood flow during a stop. Three runs were made, with one run set at $6 \mathrm{~min}$. The examinees were two males who had not driven real trains but were fully proficient in operating the simulator. The examinees were instructed to drive according to the signals and indicators, following a timetable. The measurement instrument used was a near-infrared imaging system (OMM-3000, Shimadzu Corporation, Japan) as shown in fig. 4. Fig. 5 shows the arrangement of optical fibers and measurement positions. Numbers represent measurement channels. Twenty-two (22) channels were set in the frontal region and 22 in the occipital region, giving total of 44 channels. 


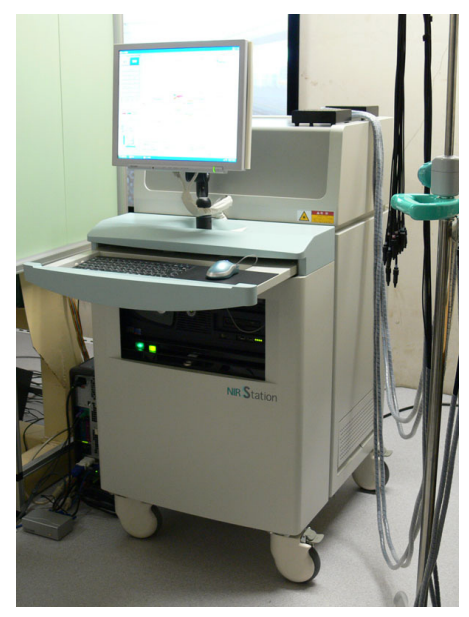

Figure 4: A near-infrared imaging system.

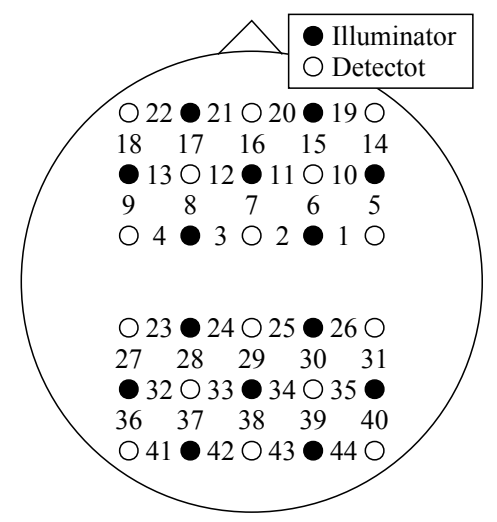

Figure 5: $\quad$ Arrangement of optical fibers and measurement positions.

\subsubsection{Measurement results}

Fig. 6 shows the results from all the channels in the frontal region, measured just after starting while the driver was in the section with 3 stations. The general trend was that the oxy-Hb concentration increased and the deoxy-Hb concentration decreased over the course of the operating time, indicating activation of the brain. Similar activation was observed in the occipital region. Furthermore, changes in hemoglobin concentration were observed in response to tasks of stopping at stations. The result of channel 7 which shows remarkable task-related changes is shown in Fig. 8. However, fNIRS signals include measurement noise and any changes in brain activities which are not related to the driving operation. To compare the changes in brain activity with individual driving operations, it is necessary to extract the task-related signal. We propose an analysis method using a wavelet-based multi-resolution analysis. 


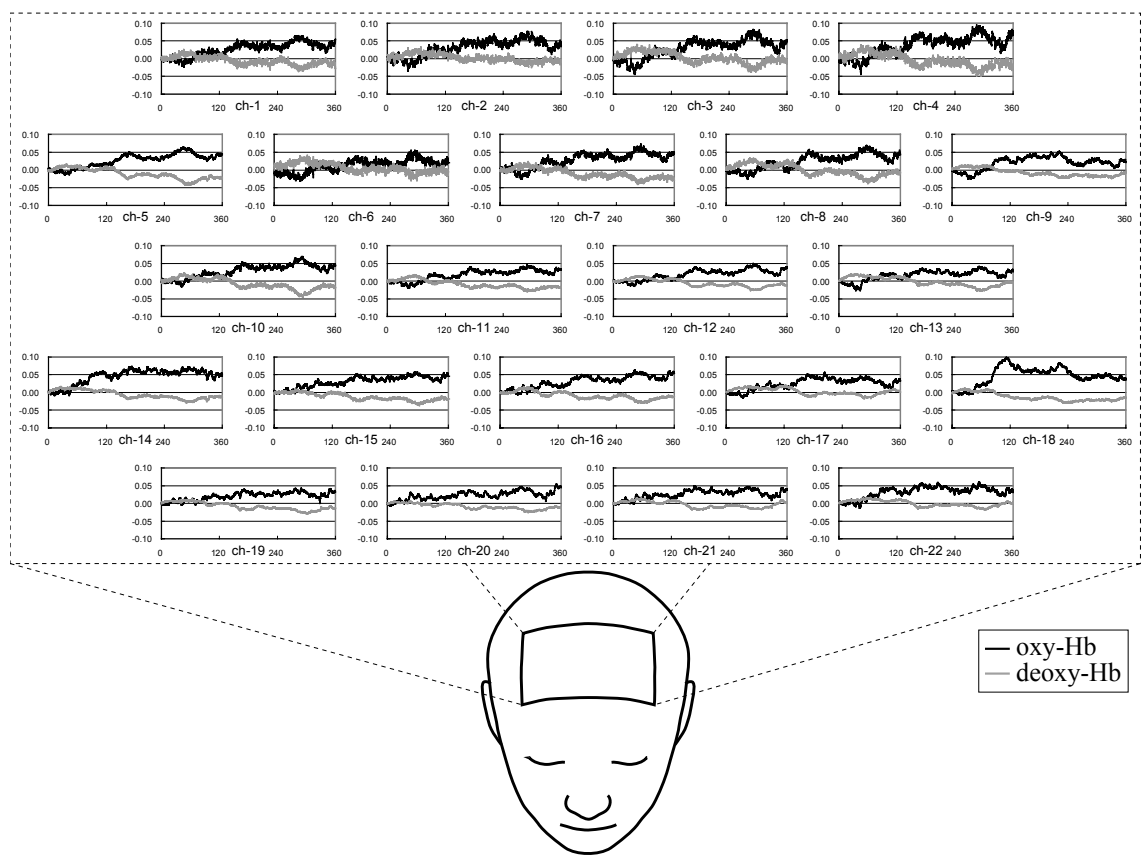

Figure 6: Changes in hemoglobin concentration in frontal.

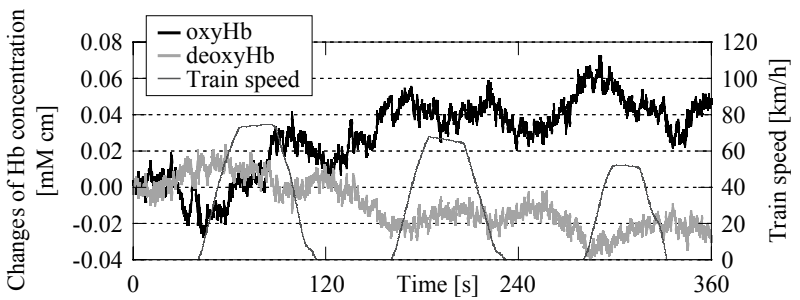

Figure 7: Changes in hemoglobin concentration on channel 7 and train speed.

\section{Decomposition and reconstruction of fNIRS signal by wavelet-based multi-resolution analysis}

\subsection{Wavelet transform}

In wavelet transform [2], a signal $S(t)$ is described by shifting and scaling a small wave, $\psi(t)$, called a mother wavelet. The signal is analyzed on the basis of the mother wavelet transform. A continuous wavelet transform (CWT) is given by:

$$
\left(W_{\psi} S\right)(a, b)=\int_{-\infty}^{\infty} \frac{1}{\sqrt{a}} \overline{\psi\left(\frac{t-b}{a}\right)} S(t) d t
$$


$\psi((t-a) / b)$ shifts the time (phase) by $b$ and makes the frequency $1 / a$. This continuous wavelet transform, however, requires a considerable amount of computation, and information is redundant. Therefore, by making $a=2^{m}$ and $b=2^{m} n$ in eqn (1), we obtain:

$$
D_{m, n}=\int_{-\infty}^{\infty} S(t) \psi_{m, n}(t) d t
$$

where:

$$
\psi_{m, n}(t)=2^{-m / 2} \psi\left(2^{-m} t-n\right)
$$

which is called a discrete wavelet transform (DWT). This discrete wavelet transform is characterized by more efficient translation of signals.

\subsection{Multi-resolution analysis}

Multi-resolution analysis (MRA) decomposes a signal into a number of components at different resolution by using the discrete wavelet transform. A time history $S(t)$ is decomposed into some detailed (high-frequency) components and an approximated (low-frequency) component. The original signal $S(t)$ given by:

$$
S(t)=S_{m 0}(t)+\sum_{m=-\infty}^{m 0} d_{m}(t)
$$

where $d_{m}$ are detailed components:

$$
d_{m}(t)=\sum_{n=-\infty}^{\infty} D_{m, n} \psi_{m, n}(t)
$$

In the wavelet transform, the choice of a mother wavelet $\psi(t)$ is important. We employed Daubechies wavelet [1], which is orthonormal base and compactly supported wavelet. The vanishing moments of Daubechies wavelet can be changed by index $N$. We decided to use a relatively high-order generating index, $N=7$ (fig. 8).

\subsection{Decomposition and reconstruction of fNIRS signals}

Fig. 9 shows the result in multi-resolution analysis of the decomposition level of ten (10) for oxy-Hb signal on channel 7 (shown in Fig. 7). Fig. 8 indicates that the trend of all the operation subjects was extracted in the approximation component $\left(a_{10}\right)$, and that other changes were decomposed into the detail components. Components $d_{1}-d_{3}$ had comparatively large amplitudes, which we considered were caused by heartbeat-related blood flow and measurement noise. Large changes were observed in components $d_{9}$ and $d_{10}$. These were considered to be task-related changes, because the repetition interval of the starting and stopping at stations is $120 \mathrm{~s}$, which is in the frequency band of component $d_{10}$. We added components $d_{8}, d_{9}$ and $d_{10}$ to reconstruct. 


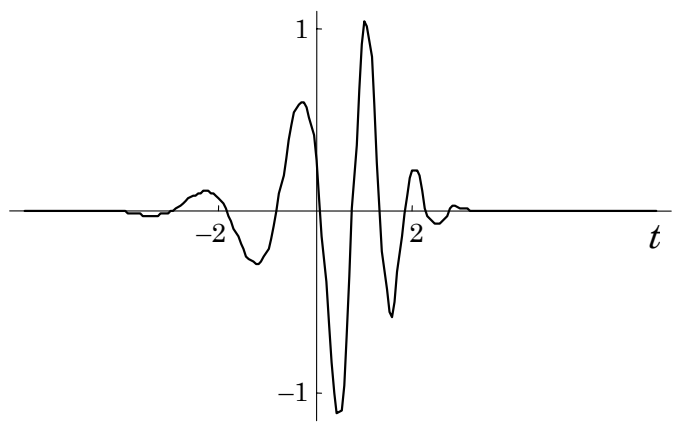

Figure 8: Daubechies wavelet $(N=7)$.
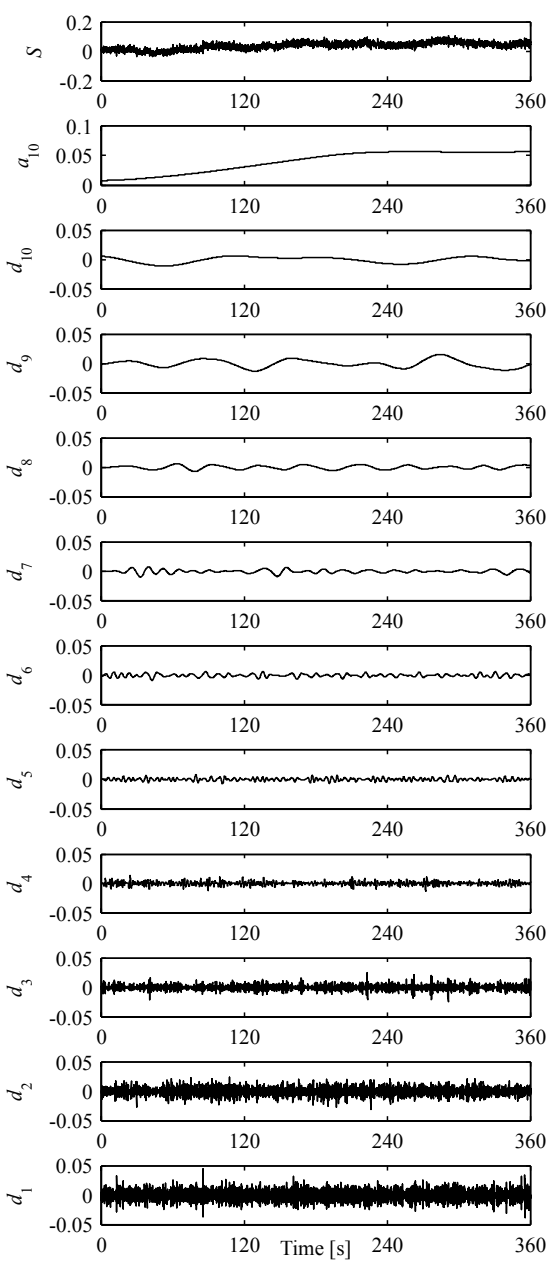

Figure 9: Multi-resolution analysis of oxy-Hb signal on channel 7. 
Fig. 10 shows the comparison of the reconstructed signals on channel 7 (shown in Fig. 7) and train speed. oxy-Hb concentration was highest when departing from stations, and deoxy-Hb showed the opposite trend. This confirmed activation of the brain during stops.

After the reconstruction for every measured channel, we created the functional brain images in frontal. The results are shown in fig. 11. We consider this imaging is very effective in evaluating driving behavior.

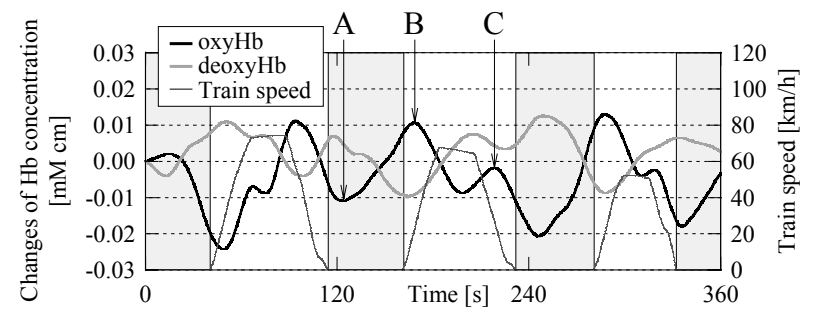

Figure 10: Reconstructed signals of changes in hemoglobin concentration on channel 7.

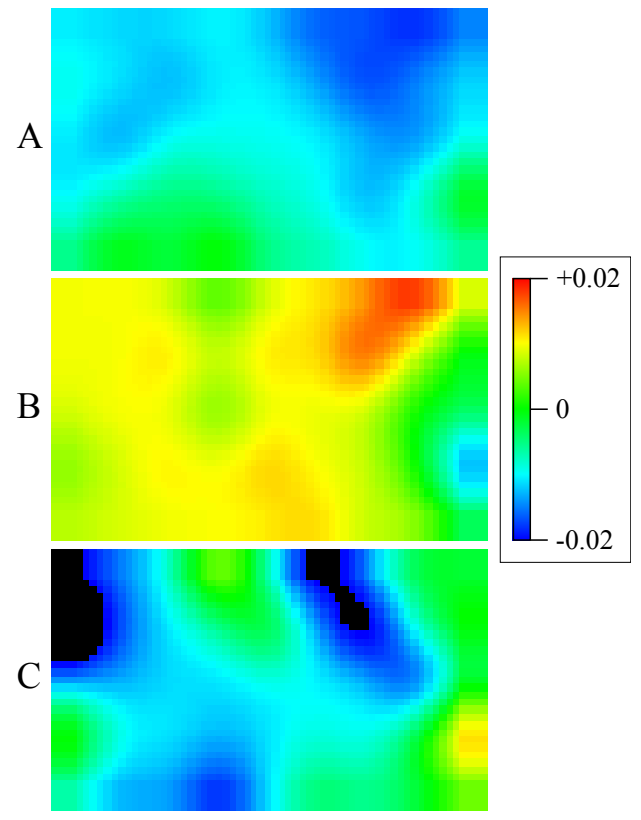

Figure 11: Functional brain imaging in frontal.

\section{Conclusions}

We measured brain function by using functional near-infrared spectroscopy (fNIRS) during simulator operation. The results show that oxy-Hb concentration 
increased and deoxy-Hb decreased with driving, and that train driving activated the brain. Additionally, multi-resolution analysis using wavelet transform is useful for evaluating brain activity in response to individual driving operations.

We are planning a future investigation into the physiological evaluation of changes in cerebral blood flow by making simultaneous measurements and comparisons of fMRI, brain waves and heartbeat.

\section{References}

[1] Daubechies, I., Orthonormal bases of compactly supported wavelets, Communications on Pure and Applied Mathematics, 41(7), pp. 909-996, 1988

[2] Daubechies, I., Ten Lectures on Wavelets, CBMS-NSF Regional Conference Series In Applied Mathematics: Society for Industrial and Applied Mathematics, (61), 1992

[3] Eda, H. et al, Multi-channel time-resolved optical tomographic imaging system, Rev Sci Instrum, (70), pp. 3595-3602, 1999

[4] Jöebsis, FF., Noninvasive, infrared monitoring of cerebral and myocardial oxygen sufficiency and circulatory parameters, Science, (198), pp. 1264 1267, 1977

[5] Kobayashi, A. \& Miyamoto, Y., In-flight cerebral oxygen status: continuous monitoring by near-infrared spectroscopy, Aviat Space Environment Med, (71), pp. 177-183, 2000

[6] Villringer, A. \& Chance, B., Non-invasive optical spectroscopy and imaging of human brain function, Trends Neurosci, (20), pp. 435-442, 1997 